\title{
El discurso docente y la pregunta como estrategia didáctica para generar reflexión crítica de contenidos
}

\author{
The teaching speech and the question as a didactic \\ strategy to promote a critical reflection on contents
}

Jaime Rosales Ma., Carmen Eugenia Universidad Tecnológica del Norte de Guanajuato

(Guadalajara - México) eugenia.jaime@utng.edu.mx

Revista Cumbres Vol.5 №2

Versión impresa ISSN 1390-9541

Versión electrónica ISSN 1390-3365

http://investigacion.utmachala.edu.ec/revistas/index.php/Cumbres 


\title{
RESUMEN
}

El trabajo que se muestra es el resultado de dos años de investigación en el contexto de las aulas del nivel Técnico Superior Universitario en el área de las TIC's, en la región nordeste del estado de Guanajuato México. Ésta abarcó tres momentos: Recuperación, Problematización e Innovación de la práctica docente, buscando mediante la aplicación de los ejes de innovación, generar en la práctica docente un camino que gestione y promueva en los estudiantes el diálogo y la criticidad, no únicamente en el ámbito escolar, sino también en el social y personal. Es a través de la investigación-acción, entendiéndola como el estudio de la situación social a fin de mejorar la acción sobre la misma, una forma de auto indagación reflexiva. A partir de la estructura de innovación-intervención se promovió en los estudiantes el avance dentro de una ruta crítica (Martínez-Otero 2010 y Fisher 2013) de los nivel 1 y 2 al nivel 3 que en términos generales significó un progreso del $80 \%$ del grupo estudiado. Los resultados son alentadores aunque éstos supondrían nuevas indagaciones y reflexiones en torno a otras estrategias que propicien la comprensión crítica; asimismo es necesario investigar sobre los propios procesos cognitivos de los estudiantes.

Palabras clave: Problematización, Ruta crítica, Investigación-acción, diálogo y criticidad.

\begin{abstract}
The work presented is the result of two years of research in a context of the Advanced Technical Degree learning centers in ICT's fields, in the northeast region of the state of Guanajuato, Mexico. This investigation ran through three stages: Recovery, Problematization and Innovation of the teaching practices with the following objective: Through the application of innovative ideas, create a route within the teaching methods to manage and promote an open dialogue and criticism between students, not only academically but socially and personally. Understanding investigation-action as a study of the social context with the purpose of improving the action in itself, it works out as a self-inquiring reflexion, which is a cyclic process in research. Starting from the innovation-intervention structure, an improvement of the critical route was promoted between the students (Martínez-Otero 2010 \& Fisher 2013) from the 1 st and 2 nd levels to the 3rd one in general terms it meant a progress of $80 \%$ of the focus group. The results are encouraging although these would involve new investigations and reflections around different strategies to foster a critical comprehension. Furthermore, it is necessary to do research and act upon the cognition processes of the students.
\end{abstract}

Keywords: Problematization, critical route, investigation-action, dialogue, criticism.

\section{Cumbres}




\section{INTRODUCCIÓN}

Somos habitantes de un mundo complejo, que resulta arduo vivir en él y para él, lo que a la vez resulta excitante y apasionante; el enfocarse a vivir un compromiso de búsqueda constante de ser la mejor persona posible, de merecer la oportunidad de vivir en este planeta que nos gestó y nos expulsó de la entraña; para ser promotores de la vida, del cuidado del cosmos, y también de ser y trascender; de transformarnos y transformar con nuestro vivir a las generaciones con las que nos involucramos en esta importantísima responsabilidad de ser mediadores en el proceso formativo de los estudiantes que llegan a estar en los espacios áulicos.

La investigación desarrollada forma parte de una doble preocupación: Recuperar y deconstruir la práctica docente, con el propósito de estar Ad hoc a las necesidades de formación de los ciudadanos del Siglo XXI y con ello fomentar en los estudiantes el desarrollo de herramientas para todos los ámbitos que los conforman y promover en ellos, con voluntad consciente, la criticidad a partir de los contenidos de las asignaturas.

El contexto y desarrollo de la investigación se sitúa en una Universidad pública de México, la cual, es un organismo público descentralizado de la administración pública estatal, con personalidad jurídica y patrimonio propios.

El subsistema de Universidades Tecnológicas en México como lo expresan (Villa Lever y Flores Crespo, 2002, p. 20) nace con los propósitos de impulsar la formación vocacional o profesional, diversificar la oferta y ofrecer estudios de nivel superior en regiones con relativa desventaja económica y social. Asimismo, con base en la decisión tomada por la Secretaría de Educación de México, al momento de la fundación de este subsistema, bajo el Modelo Francés el cual marcó las directrices para su implementación, se incrementó de 2000 horas de formación a 3000 por considerar, que los egresados del nivel medio superior mostraban una formación académica deficiente.

Volviendo a la preocupación inicial, que da como resultado la investigación sobre la propia práctica docente y la necesidad de desarrollar la criticidad en los estudiantes; es necesario también aumentar al contexto descrito en los dos párrafos anteriores, la situación en relación al desarrollo cognitivo de los estudiantes que egresan del nivel medio superior y aún en el superior, para ello sostiene:

Las operaciones del pensamiento formal deberían ser imprescindibles.

La realidad, sin embargo, entrega datos negativos (...) Investigaciones realizadas tanto en países desarrollados como en los que están en vías de desarrollo demuestran que la mayoría de los estudiantes(...) no manifiestan de manera sistemática esquemas de pensamiento formal. En el caso de Latinoamérica esto ha sido demostrado (...) también para el caso de México (Lemir, R 1983; Díaz Barriga, A 1984; Lule 1986 y Rodríguez, entre otros citado por Uribe, 1993, 4p.)

Bajo este contexto y realidad es que emerge la necesidad de poder cuestionar la propia práctica docente, tal como lo expresa (Jackson, 2013, p.15) para poder comprender lo que sucede en las aulas es necesario tratar de explicar 
cuál es el conocimiento tácito de los profesores y profesoras, o sea los constructos, principios y creencias con los que este colectivo de profesionales deciden y actúan. Esto se logra a través de actos reflexivos, con voluntad consciente y sistematización por parte del docente.

Uno de los elementos que se fueron develando en el proceso de investigación, que se está describiendo, es el pensamiento crítico, al que se abordó desde su doble dimensión: analítica y evaluativa; el cual se remonta al principio de los tiempos en la educación de los ciudadanos, tal como lo afirma Albertos (2015, p.38).

Y sigue la cita; este autor muestra tres fases en la forma en que se ha abordado el estudio e implementación en el ámbito educativo de este tipo de pensamiento:

La primera fase abordada como capacidades que podían abordarse como objetivos; En la segunda fase se dio mayor interés a los procesos de pensamiento crítico necesarios para la resolución de problemas, la toma de investigación y en la tercera fase como procesos y habilidades de pensamiento crítico en distintas situaciones en los contextos escolares y personales. Se produce un marcado interés por la transferencia de estas habilidades como medio para la meta-cognición, esperando mayor autonomía y conciencia por parte de los estudiantes.

Análogamente, el discurso y en particular el del docente emerge como unidad de análisis para el proceso de investigación ya que necesariamente éste actúa como herramienta para fomentar la reflexión. (Martínez-Otero, 2010, p.45) lo define como Peculiar praxis comunicativa que posibilita la relación interhumana y la formación [...] el discurso educativo como un entramado lingüístico que permite expresar ideas, informaciones y estados afectivos para facilitar el proceso formativo.

\section{MATERIALES Y MÉTODOS}

En la investigación que se describe en el presente documento, se pueden identificar tres grandes etapas: Recuperación y análisis de la práctica docente, la problematización de la misma y finalmente la Innovación de ésta.

Este proceso de investigación se sitúa bajo una metodología cualitativa que tuvo como propósito obtener datos descriptivos a partir de las propias palabras de la docente, que redacta el presente trabajo, y los estudiantes de ésta y de las conductas observables de ambos agentes educativos. Se buscó realizar descripciones detalladas de situaciones, eventos, personas, interacciones y comportamientos observables, incorporando la voz de los participantes, las experiencias, actitudes, creencias, pensamientos y reflexiones tal y como eran expresados por ellos.

Esta metodología se apoyó en los recursos etnográficos como lo son, los registros de observación de clase; dando paso a micro ensayos de primer y segundo nivel y con ellos recuperar y comprender la propia práctica docente,

\section{Cumbres}


como lo postula (García, A.1977, 52p) el docente que intenta recuperar su propia práctica docente necesita "Hacer que lo familiar se vuelva extraño" palabras de Erickson citadas por García .Ya que si se deja llevar por la corriente de las actividades cotidianas, nos será capaz de identificar hechos que por conocidos se pasan por alto, pero que en un proceso de investigación resulta imprescindibles para comprender lo que pasa.

Se hace necesario apuntar que la investigación se realizó durante dos años, eso significó que se abordaron dos generaciones: una del ciclo escolar 2016-2017 y otra del ciclo 2017-2018. En la etapa de recuperación se abordó con estudiantes de cuarto cuatrimestre y en la etapa de Problematización e Innovación fue con los grupos de estudiantes desde el primero, segundo y tercer cuatrimestres.

De la etapa de Recuperación y análisis de la Práctica Docente se realizaron dos registros de clase y un micro ensayo de primer y otro de segundo nivel, la población con la que se desarrolló la recuperación fue con un grupo de estudiantes del programa educativo de Sistemas Informáticos del $4^{\circ}{ }^{\circ}$ cuatrimestre.

Cabe hacer mención que, las características de estos registros etnográficos permitieron hacer el análisis sistemático del proceso educativo promovido por la docente en cuestión, con la observación de la clase objeto de estudio, "se describió lo qué se hacía, qué significaba lo que se hacía, cómo se ha llegado a ser esa docente, lo que permitió posteriormente hacer una deconstrucción para dar respuesta a cómo se pueden hacer las cosas de modo distinto". (García, A 1997, 61p.).

En la fase de recuperación, el registro contó con los elementos tales como: contexto curricular de Institución, datos generales, intencionalidad de la sesión de clase; estrategias didácticas empleadas; ubicación y distribución del aula; momentos, duración, hechos, inferencias, distribución del habla del docente y de los estudiantes.

De esta recuperación, a partir del primer registro de clase, se pudo observar que el habla de la docente, abarcaba el 57.14\% mientras en los estudiantes el $42.85 \%$; en él mimo se detectó que la docente formulaba preguntas pero, no había atención a resolverlas, existía improvisación, reiteración de ejemplos y la maestra se mantenía en un área del salón. Finalmente sobresale la estructura de las preguntas realizadas por ella, que se podían responder con un sí o con un no; o bien que reiteraban lo dicho por ella y si se adiciona la calidad de atención brindada al estudiante, pues la comprensión crítica de los contenidos y el diálogo quedaban pendientes.

Los hallazgos encontrados durante la primera etapa permitieron delimitar la línea de trabajo y dar paso a la Problematización de la práctica docente, lo que apuntó sobre el discurso docente, sobre todo que en la intención educativa explícita, o quizá en la propia filosofía educativa, se ha tenido como intencionalidad educativa, la generación de criticidad y reflexión y aprendizaje dialógico.

Así pues, en esta segunda etapa se buscó la problematización de la propia práctica a partir de integrar los seis constitutivos que conforman a la docen- 
cia, de acuerdo a la metodología propuesta por Bazdesch (2000): el contexto, el modelo, la subjetividad de los participantes, la intersubjetividad, el proceso cognoscitivo y el contenido.

Se realizó un segundo registro de sesión de clase y con base en los datos y análisis de éste, se procedió a la construcción de la arqueología de la propia práctica, como se muestra en la siguiente imagen: Las cantidades en cada figura representan los momentos que ocuparon cada constitutivo en el desarrollo de clase grabada.

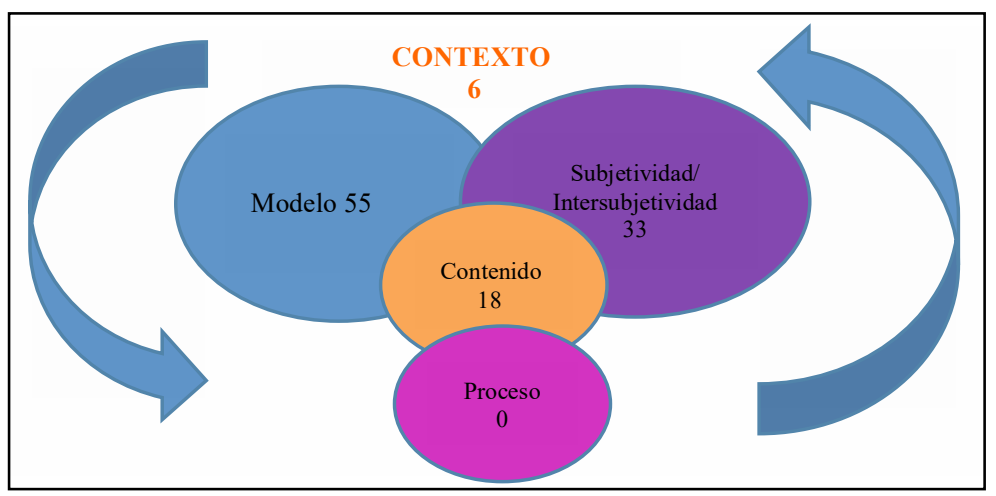

Figura 1. Arqueología de la Práctica docente.

En esta segunda etapa de la investigación se identificó una importante intersección entre discurso (subjetividad/intersubjetividad) y el Modelo o revestimiento, como las unidades de observación con las que se continuaría en la investigación, respaldado esto por los hallazgos encontrados durante la estructuración de la arqueología de la práctica docente y por supuesto, por el estado del arte o conocimiento.

De tal manera se estructuró la siguiente pregunta:

¿Qué elementos estructuran el discurso de maestra de las asignaturas de formación sociocultural de los programas educativos de Sistemas Informáticos, Multimedia y Comercio Electrónico y Redes y Telecomunicaciones de la Universidad Tecnológica y en qué medida ese discurso favorece la comprensión crítica de los contenidos?

Para dar paso a la tercera etapa de la investigación es importante retomar la filosofía educativa propia, pues permite encarar de forma consciente la intencionalidad educativa y hacer las innovaciones que correspondan; para ello se respalda nuevamente en una educación que haga frente a la criticidad y la metacognición, una que permita desarrollar una visión democrática, autoreflexiva y cuestionadora.Galindo (2015) expresa que:

La educación para la democracia, que va desde la niñez temprana hasta la universidad, está articulada por tres ejes: la argumentación socrática, el conocimiento de los otros (dentro y fuera del propio país) que nos hace "ciudadanos del mundo", y la imaginación narrativa. Esta

\section{Cumbres}


educación, como decía, se contrapone a la educación con fines de lucro, caracterizada por la pasividad de los estudiantes.(p. 113).

Así pues, la fase de innovación se inicia con un profundo cuestionamiento sobre ¿qué innovar, para qué y/o por qué?; la estructuración de una ruta crítica y la intervención en tres sesiones de clase.

Con respecto al primer cuestionamiento (Barraza,2001. 15p.) afirma que la innovación es un proceso que involucra la selección, organización de elementos involucrados [...] la enseñanza [...] debe ser desarrollada en una manera deliberada y sistemática con el objetivo de lograr un cambio duradero.

En consecuencia con lo problematizado sobre el quehacer docente que son el discurso y el modelo docentes permite construir como ejes fundamentales para la innovación. Respecto del primero se hace conveniente recordar el respaldo teórico metodológico que dan a esta investigación Martínez-Otero (2010); Fisher (2013) y Carretero (2004). El primer autor permite conceptualizar del discurso como una praxis comunicativa encaminada a promover el desarrollo personal del educando. Para Fisher (2013) es un medio de centrar atención, mantener el diálogo, estimular el pensamiento y la criticidad exige estar dispuestos a someter las ideas propias y ajenas. Finalmente para Carretero(2004) las preguntas son instrumentos de mediación.

El siguiente punto por mencionar, es la estructuración de una ruta crítica defina con base en UcMas (2005):

Un instrumento conceptual específico que desmenuza las intenciones del docente y las hace operables en un camino susceptible de monitorear para observar el nivel de logro en los alumnos. Ésta permite ubicar a los estudiantes en los distintos niveles, momentos o fases del conjunto de tareas que se desea desarrollen éstos, a partir de una intencionalidad educativa definida.

Tabla 1: A La ruta crítica construida para la innovación-investigación

\begin{tabular}{|c|c|c|c|}
\hline $\begin{array}{l}\text { Discurso del } \\
\text { Maestro }\end{array}$ & Características & Impacto en el estudiante & Ruta Crítica \\
\hline Dimensión & $\begin{array}{l}\text { Terminología técnica } \\
\text { y científica, según las } \\
\text { materias o asignaturas } \\
\text { [Inclusión de datos [...] } \\
\text { Abundancia de conceptos } \\
{[\ldots]}\end{array}$ & $\begin{array}{l}\text { Repite la lección, sin } \\
\text { reflexión, ni compren- } \\
\text { sión. } \\
\text { Memorización, se centra } \\
\text { en los resultados }\end{array}$ & $\begin{array}{l}\text { Nivel 1: Escucha para } \\
\text { aprender: } \\
\text { Sienta las bases para } \\
\text { aprender } \\
\text { Participa e Indaga } \\
\text { Toma notas, Mira hacia } \\
\text { adelante } \\
\text { hacia quién habla }\end{array}$ \\
\hline $\begin{array}{l}\text { Dimensión } \\
\text { Afectiva }\end{array}$ & $\begin{array}{l}\text { Diálogo con alumnos } \\
\text { Actitud favorecedora } \\
\text { Carece de homogeneidad } \\
\text { Expresión de estados de } \\
\text { ánimo } \\
\text { Palabras de afecto [...] } \\
\text { Valoración } \\
\text { positiva de los alumnos. } \\
\text { [...]Predomina } \\
\text { la función expresiva. }\end{array}$ & $\begin{array}{l}\text { Responde ante los medios } \\
\text { audiovisuales, imágenes } \\
\text { pero es intolerante al dis- } \\
\text { curso lógico-racional. } \\
\text { Se involucran a través de } \\
\text { la anécdota, las expresio- } \\
\text { nes coloquiales [...] nivel } \\
\text { de } \\
\text { conceptualización de la } \\
\text { vida cotidiana. }\end{array}$ & $\begin{array}{l}\text { Nivel 2: Pensar y recor- } \\
\text { dar } \\
\text { Preguntas básicas del } \\
\text { contexto } \\
\text { cotidiano }[\ldots]\end{array}$ \\
\hline
\end{tabular}




\begin{tabular}{|c|c|c|c|}
\hline $\begin{array}{l}\text { Dimensión } \\
\text { motivacional y } \\
\text { social }\end{array}$ & $\begin{array}{l}\text { Presentación de conteni- } \\
\text { dos } \\
\text { nuevos. Discurso jerar- } \\
\text { quizado } \\
\text { y coherente. Empleo ha- } \\
\text { bitual } \\
\text { de ejemplos [...] Lenguaje } \\
\text { animado por imágenes } \\
{[\ldots]}\end{array}$ & $\begin{array}{l}\text { Los estudiantes } \\
\text { motivados, responden al } \\
\text { estímulo intelectual. } \\
\text { Intercambio verbal, } \\
\text { escucha activa, estruc- } \\
\text { tura } \\
\text { y muestra argumentos. } \\
\text { Establece conexiones }\end{array}$ & $\begin{array}{l}\text { Nivel 3: Discusión } \\
\text { Intercambio verbal y } \\
\text { sistemático [...] mediante } \\
\text { la contrastación de argu- } \\
\text { mentos y } \\
\text { evidencia, afirmaciones, } \\
\text { cuestionamientos } \\
\text { conflicto cognitivo;[...] } \\
\text { Investiga presupuestos, } \\
\text { Busca razones y } \\
\text { evidencias. }\end{array}$ \\
\hline $\begin{array}{l}\text { Dimensión } \\
\text { Ética }\end{array}$ & $\begin{array}{l}\text { Organización axiológica } \\
\text { de la realidad. } \\
\text { [...]Técnicas diversas: es- } \\
\text { tudio } \\
\text { de casos, discusiones } \\
\text { Práctica de acciones mo- } \\
\text { rales } \\
\text { para favorecer hábitos } \\
\text { positivos. }\end{array}$ & $\begin{array}{l}\text { Escucha bien. } \\
\text { Participa; Indaga; } \\
\text { Recuerda para dar } \\
\text { continuidad; Investiga } \\
\text { Da puntos de vista; } \\
\text { Pregunta, comprueba. }\end{array}$ & $\begin{array}{l}\text { Nivel 4: Diálogo } \\
\text { Implica a dos o más per- } \\
\text { sonas } \\
\text { que se escuchan unas a } \\
\text { otras, } \\
\text { comparten ideas y tienen } \\
\text { encuentra los distintos } \\
\text { puntos } \\
\text { de vista, Líneas coheren- } \\
\text { tes } \\
\text { de pensamiento y de in- } \\
\text { dagación }\end{array}$ \\
\hline
\end{tabular}

Fuente: Construcción investigadora con base en Martínez-Otero (2010); Fischer (2013)

Una vez estructurada la ruta crítica se realizó un registro de clase para identificar con base en ella, en qué nivel se encontraban los estudiantes y estructurar las sesiones con el propósito de hacer la innovación en la propia práctica.

Se considera pertinente, recordar que toda la metodología se fundamenta en la perspectiva de la Investigación-Acción en donde la docente se ubica, como lo refiere Eliott (1990) citado por Torres. (2017, p. 66.) en "el centro de la investigación a través del crecimiento del currículo y se perfecciona mediante la práctica reflexiva en el aula y su investigación".

El propósito general "Propiciar en los estudiantes el intercambio verbal y sistemático, mediante el uso de la pregunta como estrategia didáctica detonadora e innovadora para la contrastación de argumentos y evidencias, afirmaciones, presupuestos, razones y evidencias que los lleve a la comprensión crítica de los contenidos".

La pregunta de innovación quedo construida de la siguiente manera:

¿A través de qué estilos de preguntas (como estrategia didáctica), implementadas en el proceso de aprendizaje, el estudiante de los programas educativos del área de Tics de la Universidad Tecnológica del Norte de Guanajuato puede transitar de un nivel de escucha para aprender (nivel 1) y Pensar y recordar (nivel 2) hacia la Discusión (nivel 3)?

En esta fase de la investigación se llevaron a cabo cuatro sesiones de clase: Una para realizar la ubicación de los estudiantes en el nivel o niveles en los que se encontraban con respecto a la ruta crítica ya descrita; y tres sesiones de clase para el proceso de innovación; Para lograr el impacto en el modelo o revestimiento, así como en los modelos conversacionales en el aula. Se diseñaron los planes de clase, bajo estrategias didácticas que permitieron

\section{Cumbres}


descentrar la figura del docente: Estrategias colaborativas; Estrategias Lúdicas y la Técnica expositiva, por parte de la docente y los estudiantes. Y la estructura de preguntas (como estrategia didáctica)

Antes de describir los resultados obtenidos es propicio mencionar que en al registro de clase se adicionaron nuevas columnas para el momento del análisis de éste: Una referida a los niveles de la ruta crítica para cada momento de la clase y dos más que hacen referencia a los niveles de intersubjetividad y otra a las operaciones cognitivas realizadas por los estudiantes por cada momento de clase.

\section{RESULTADOS}

En el presente apartado se muestran las evidencias que muestran el avance logrado con la intervención-innovación. Enseguida el análisis de dichas evidencias de aprendizaje.

La siguiente tabla muestra la colocación de los estudiantes en la ruta crítica.

Tabla 2. Fases de ruta crítica y ubicación de los estudiantes

\begin{tabular}{|c|c|c|c|c|}
\hline Momento & \multicolumn{4}{|c|}{ Nivel de ruta crítica identificado } \\
\hline \multirow{2}{*}{$\begin{array}{l}\text { Desarrollo } \\
\text { de temática } \\
\text { y cierre }\end{array}$} & $\begin{array}{l}\text { Escucha para } \\
\text { aprender (Nivel 1) }\end{array}$ & $\begin{array}{l}\text { Pensar y Recordar } \\
\text { (Nivel 2) }\end{array}$ & Discusión (Nivel 3) & Diálogo (Nivel 4) \\
\hline & $\begin{array}{l}\text { Terminología téc- } \\
\text { nica, repite ideas } \\
\text { clave, abundancia } \\
\text { de conceptos, } \\
\text { Repite la lección, } \\
\text { escucha para } \\
\text { aprender, toma } \\
\text { notas, Mira hacia } \\
\text { adelante, hacia } \\
\text { quien habla. }\end{array}$ & $\begin{array}{l}\text { Actitud favore- } \\
\text { cedora diálogo, } \\
\text { Expresión de } \\
\text { estados de ánimo, } \\
\text { responde ante los } \\
\text { medios audiovisua- } \\
\text { les, expresiones } \\
\text { coloquiales y nive- } \\
\text { les de conceptua- } \\
\text { lización de la vida } \\
\text { cotidiana. Solicita } \\
\text { información.. }\end{array}$ & $\begin{array}{l}\text { Estudiante moti- } \\
\text { vado, responden al } \\
\text { estímulo intelectual, } \\
\text { intercambio verbal, } \\
\text { escucha activa, } \\
\text { estructura y mues- } \\
\text { tra argumentos. } \\
\text { Establece conexio- } \\
\text { nes, Contrasta Ar- } \\
\text { gumentos, conflicto } \\
\text { cognitivo, busca } \\
\text { razones. }\end{array}$ & $\begin{array}{l}\text { Escucha bien, inda- } \\
\text { ga, recuerda para } \\
\text { dar continuidad, } \\
\text { Investiga, elabora } \\
\text { razonamientos, } \\
\text { da puntos de } \\
\text { vista, pregunta, } \\
\text { comprueba, Líneas } \\
\text { coherentes de } \\
\text { pensamiento e } \\
\text { indagación. }\end{array}$ \\
\hline
\end{tabular}

De acuerdo con los datos antes expuestos se encontraron los estudiantes en la ruta crítica, predominantemente en Escucha para aprender (nivel 1) y Pensar y Recordar (Nivel 2). El tema revisado en la sesión grabada fue los recursos materiales, afectivos, cognitivos, sociales con los que contaban para la estructuración de un Plan y Proyecto de Vida y Carrera. Una vez que se realizaron las tres sesiones de Innovación se pretendió que la mayoría de los estudiantes pudieran transitar al nivel de Discusión (Nivel 3).

Con base en los datos expuestos en la Tabla 3 indican el tránsito logrado de los estudiantes, ya que se pasó de nivel de supuestos a niveles de certezas y acciones a través de la construcción de sus respuestas dadas, un intercambio verbal y sistemático, además sustentan sus respuestas, dan razones. Con respecto los procesos cognitivos dan cuenta de inteligibilidad y verificación. Los registros que respaldan estos resultados hablan que el $90 \%$ de los estudiantes durante el desarrollo del rally logran el tránsito en los niveles de la ruta crítica. 
Tabla 3. Resultados de los tres momentos de Innovación

\begin{tabular}{|c|c|c|c|c|c|c|c|c|c|c|}
\hline \multirow{2}{*}{$\begin{array}{l}\text { Momen- } \\
\text { tos/Te- } \\
\text { mas }\end{array}$} & \multirow{2}{*}{$\begin{array}{l}\text { Fase de } \\
\text { u b i c a - } \\
\text { ción }\end{array}$} & \multicolumn{3}{|c|}{ Primera sesión } & \multirow{2}{*}{\multicolumn{3}{|c|}{$\begin{array}{l}\text { Segunda sesión Innova- } \\
\text { ción }\end{array}$}} & \multirow{3}{*}{\multicolumn{3}{|c|}{$\begin{array}{l}\text { Tercera sesión } \\
\text { Innovación }\end{array}$}} \\
\hline & & $\begin{array}{l}\text { Nivel } \\
\text { Logra- } \\
\text { do }\end{array}$ & $\begin{array}{l}\text { Inter } \\
\text { Subje- } \\
\text { tividad }\end{array}$ & $\begin{array}{l}\text { Pro- } \\
\text { ceso } \\
\text { cog- } \\
\text { niti- } \\
\text { vo }\end{array}$ & & & & & & \\
\hline $\begin{array}{l}\text { Recursos } \\
\text { para la } \\
\text { f or m a - } \\
\text { ción de } \\
\text { un plan } \\
\text { de vida }\end{array}$ & $\begin{array}{l}\text { Nivel } 1 \\
\text { y } 2\end{array}$ & & \multirow[t]{2}{*}{\begin{tabular}{|l} 
Nivel \\
Logra- \\
do
\end{tabular}} & $\begin{array}{l}\text { Inter- } \\
\text { sub } \\
\text { jetivi- } \\
\text { dad }\end{array}$ & $\begin{array}{l}\mathrm{P} r \mathrm{r}- \\
\mathrm{c} \text { e s o } \\
\text { cogni- } \\
\text { tivo }\end{array}$ & & & \\
\hline $\begin{array}{l}\text { La cohe } \\
\text { sión so- } \\
\text { cial }\end{array}$ & & $\begin{array}{l}\text { Nivel } \\
3\end{array}$ & $\begin{array}{l}\text { Certe } \\
\text { zas y } \\
\text { accio- } \\
\text { nes }\end{array}$ & $\begin{array}{l}\text { Inte- } \\
\text { ligi- } \\
\text { bili- } \\
\text { dad; } \\
\text { veri- } \\
\text { ficar }\end{array}$ & & & & $\begin{array}{l}\text { Nivel } \\
\text { logra- } \\
\text { do }\end{array}$ & $\begin{array}{l}\text { Inter } \\
\text { subjeti- } \\
\text { vidad }\end{array}$ & $\begin{array}{l}\text { Proce- } \\
\text { so cog- } \\
\text { nitivo }\end{array}$ \\
\hline $\begin{array}{l}\text { Lideraz- } \\
\text { go }\end{array}$ & & & & & $\begin{array}{l}\text { Nivel } \\
3\end{array}$ & $\begin{array}{l}\text { C e r - } \\
\text { tezas } \\
\text { y ac- } \\
\text { C i o - } \\
\text { nes }\end{array}$ & $\begin{array}{l}\text { Inteli- } \\
\text { gibili- } \\
\text { dad y } \\
\text { verifi- } \\
\text { cación }\end{array}$ & $\begin{array}{l}\text { Nivel } \\
3\end{array}$ & $\begin{array}{l}\text { Certe- } \\
\text { zas y } \\
\text { accio- } \\
\text { nes }\end{array}$ & $\begin{array}{l}\text { Inteli- } \\
\text { gibili- } \\
\text { dad y } \\
\text { verifica } \\
\text { ción }\end{array}$ \\
\hline
\end{tabular}

En la segunda intervención, se emplearon técnicas expositivas y estrategias lúdicas que también sostienen el tránsito dentro de la ruta crítica y los niveles de subjetividad logrado (certezas y acciones), la docente-investigadora consideró al término de sesión, que pudiera existir un retroceso, pues se redujo al $80 \%$ por las respuestas y participación dadas por los estudiantes; esto llevó a una tercera sesión con la finalidad de profundizar en los resultados. De esta última se logró mantener más del 90\% de los estudiantes en el nivel 3 de la ruta crítica y en el nivel de certezas y acciones, así como en inteligibilidad y verificación.

Si bien, tres estudiantes del grupo revelan un tránsito hacia el nivel 4 de la ruta crítica y niveles de evaluación en sus procesos cognitivos, esto representó apenas el 10\% de la población con la que se desarrolló el proceso de innovación.

\section{CONCLUSIONES}

El efecto logrado es esperanzador, mediante la aplicación de los ejes de innovación, se logró propiciar en lo estudiantes el intercambio verbal y sistemático (las evidencias escritas lo indican así) y esto promueve en los estudiantes la comprensión crítica de los contenidos. El reto cognitivo es seguir desentrañando cada constitutivo, en virtud del valor intrínseco que aporta frente a la práctica docente, no hay final que este proceso se visualiza como

\section{Cumbres}


un gran espiral que continúe trabajando en la promoción de pensamientos, aprendizajes de docentes y discentes ¿̇s posible que la cultura del docente y la de los estudiantes queden intactas; no es por ello que se destaca en este encuentro dos culturas o cien o mil culturas que se tocan y que en mucho se transforman.

Finalmente se asume que queda mucho camino por resolver, también por indagar; precisar la planeación de las actividades docentes, seguir trabajando en la construcción de preguntas a partir de necesidades específicas.

\section{REFERENCIAS BIBLIOGRÁFICAS}

Albertos, D. (2015). Diseño, Aplicación y evaluación de un programa educativo basado en competencias científicas para el desarrollo del pensamiento crítico (Tesis Doctoral)Universidad Autónoma de Madrid. España. Consultado de: https:// dialnet.unirioja.es/servlet/tesis?codigo $=48044$

Barraza,A. (2013). ¿Cómo elaborar proyectos de innovación educativa? .Durango. México: Universidad Pedagógica de Durango.

Bazdresch, M. (2000). Vivir la educación, transformar la práctica. Guadalajara. México:Ediciones SEP-Jalisco.

Carretero, A. (2004). El discurso contingente como herramienta pedagógica para favorecer la co-construcción de la docencia en la clase de alemán/LE para adultos en el nivel de principiantes (tesis doctoral), Universidad de Barcelona. España Consultado de https://dialnet.uniroja.es/servlet/tesis?codigo $=6292$

Fisher, R. (2013). Diálogo creativo hablar para pensar en el aula. Madrid. España; Edición Morta[Versión en línea] consultado de:http://site.ebrary.com. ezproxy.uveg.edu.mx/lib/uvegsecsp/detail.action?docID=10820492

Galindo, L. (2015). Lo relativo y lo universal en la defensa de las humanidades para la democracia. En revista Literatura: teoría, historia, crítica consultado de http:// dx.doi.org/10.15446/lthc.v17n2.51279

García, A., Campechano, J., Minakata, A. y Sanudo, L. (1987). La instrumentación metodológica en la recuperación de la práctica docente. Guadalajara. México: Unidad editorial del Gobierno del Estado de Jalisco.

Jackson, W. (2013). La Vida en las Aulas. Madrid. España: Morata.

Martínez-Otero, V. (2010). El discurso educativo y la mejora del perfil docente, discente e institucional. Anuario del Doctorado en Educación: Pensar la Educación, [S.1.], n. 4, p. 43-59. ISSN 224-8594. Disponible en: http://erevistas. saber.ula.ve/index.php/anuariodoctoradoeducacion/article/view/3866

Torres, E.F. (2017). Innovación en la enseñanza de la Estadística. En Diálogos Educativos. Vol 17. Recuperado de: http://www.dialogoseducativos.cl/revistas/ n32torres.

UcMas, L (2005).Innovación, intervención, transformación. A propósito de la práctica educativa. En Revista Práctica No.8.

Uribe, M. (2003). El desarrollo del pensamiento formal y la docencia universitaria .En Peiles Educativos.No 60. Recuperado de: http://www.redalyc.org/articulo.oa?id=13206009. 
Villalever, L. y Flores P. (2002) Las universidades tecnológicas mexicanas en el espejo de los institutos universitarios de tecnología franceses. En Revista Mexicana de Investigación Temática, Vol.7. Recuperado de: http://www.redalyc. org/articulo.oa?id=14001403.

\section{Cumbres}

